\title{
Doppler shift oscillations from a hot line observed by IRIS
}

\author{
D. $\mathrm{Li}^{1,2,3}$, Z. J. Ning ${ }^{1}$, Y. Huang ${ }^{1}$, N.-H. Chen ${ }^{4}$, Q. M. Zhang ${ }^{1}$, Y. N. Su${ }^{1}$, and W. Su${ }^{1,2}$ \\ ${ }^{1}$ Key Laboratory of Dark Matter and Space Astronomy, Purple Mountain Observatory, CAS, \\ Nanjing 210008, China \\ ${ }^{2}$ Key Laboratory of Modern Astronomy and Astrophysics (Nanjing University), Ministry of \\ Education, Nanjing 210023, China \\ ${ }^{3}$ CAS Key Laboratory of Solar Activity, National Astronomical Observatories, Beijing 100012, \\ China \\ ${ }^{4}$ Korea Astronomy and Space Science Institute, Daejeon 34055, Korea
}

\begin{abstract}
We present a detailed investigation of the Doppler shift oscillations in a hot loop during an M7.1 flare on 2014 October 27 observed by the Interface Region Imaging Spectrograph. The periodic oscillations are observed in the Doppler shift of Fe XXI $1354.09 \AA$ $(\log T \sim 7.05)$, and the dominant period is about 3.1 minutes. However, such 3.1-min oscillations are not found in the line-integrated intensity of Fe XXI 1354.09 A, AIA EUV fluxes, or microwave emissions. SDO/AIA and Hinode/XRT imaging observations indicate that the Doppler shift oscillations locate at the hot loop-top region ( $\geq 11 \mathrm{MK})$. Moreover, the differential emission measure (DEM) results show that the temperature is increasing rapidly when the Doppler shift oscillates, but the number density does not exhibit the corresponding increases nor oscillations, implying that the flare loop is likely to oscillate in an incompressible mode. All these facts suggest that the Doppler shift oscillations at the shorter period are most likely the standing kink oscillations in a flare loop. Meanwhile, a longer period of about 10 minutes is identified in the time series of Doppler shift and line-integrated intensity, GOES SXR fluxes and AIA EUV light curves, indicating the periodic energy release in this flare, which may be caused by a slow mode wave.
\end{abstract}

Subject headings: line: profiles — Sun: flares — Sun: oscillations — Sun: UV radiation

\section{Introduction}

Oscillations have been commonly detected in the solar atmosphere, and they are often characterized by the regular peaks or the periodic motions. The oscillations can be identified as the

Correspondence should be sent to: lidong@pmo.ac.cn, ningzongjun@pmo.ac.cn 
brightness fluctuations from the total fluxes in a broad wavelength, such as microwave emissions (Melnikov et al. 2005; Tan \& Tan 2012), extreme-ultraviolet and ultraviolet (EUV/UV) passbands (De Moortel et al. 2002; Ning 2017), soft and hard X-ray (SXR/HXR) channels (Lipa 1978; Li \& Gan 2008; Ning 2014). On the other hand, the oscillations can also be identified as the spatial displacement movements in the imaging observations (Aschwanden et al. 1999; Shen \& Liu 2012; Shen et al. 2013), as well as in the spectroscopic observations such as the properties of the line profiles - Doppler shift, line intensity and width (Tian et al. 2011; Li \& Zhang 2015; Li et al. 2015a; Zhang et al. 2016a). The current discovered oscillatory periods can range from tens of milliseconds $\left(10^{-2} \mathrm{~s}\right)$ to thousands of seconds $\left(10^{3} \mathrm{~s}\right)$ in the multi-wavelength observations from radio through EUV/UV to X-ray emissions (e.g., Schrijver et al. 2002; Inglis \& Nakariakov 2009; De Moortel \& Nakariakov 2012). And the oscillations within multi-periods have also been detected in the same event (Zimovets \& Struminsky 2010; Yang \& Xiang 2016; Li et al. 2017a).

Over decades, the oscillations in coronal or flare loops are particularly investigated (e.g., Nakariakov et al. 1999; Kliem et al. 2002; Goddard \& Nakariakov 2016; Yuan \& Van Doorsselaere 2016a). Using the imaging observations from the Transition Region and Coronal Explorer (TRACE), the loop oscillations are first detected as the spatial displacement oscillations in solar corona (see., Aschwanden et al. 1999; Nakariakov et al. 1999), and details are subsequently reported by Aschwanden et al. (2002) and Schrijver et al. (2002). In the Solar Dynamics Observatory (SDO) era, these oscillations are further studied in flare or coronal loops (e.g., Wang et al. 2012; Nisticò et al. 2013; Yuan \& Van Doorsselaere 2016b). Currently, two regimes are found: one is the rapidly damping oscillations within only a few cycles and the other is decayless oscillations. The amplitudes of the rapidly damping oscillations are usually large and decay with time (Aschwanden et al. 1999; Nakariakov et al. 1999; Zimovets \& Nakariakov 2015). While those decayless oscillations with small amplitudes can last for tens of cycles (Nisticò et al. 2013; Anfinogentov et al. 2015). The loop oscillations can also be observed in the warm and hot emission lines which formed in solar corona or flares, such as Fe XIX $1118 \AA$ and Fe XXI $1354 \AA$ from SOHO/SUMER (Wang et al. 2002, 2003), Ca XIX $3.1781 \AA$ and S xv 5.0379 $\AA$ from YOHKOH/BCS (Mariska 2005, 2006), Fe XII $195.12 \AA$ and Fe XIII $202.04 \AA$ from Hinode/EIS (Tian et al. 2011), Fe XXI $1354.09 \AA$ from IRIS (Tian et al. 2016).

Those oscillations detected in coronal or flare loops are often explained by MHD waves (Nakariakov \& Melnikov 2009; De Moortel \& Nakariakov 2012), such as fast kink waves (Su et al. 2012a; Nisticò et al. 2013; Yuan et al. 2016), slow magnetoacoustic waves (Ofman \& Wang 2002; Wang et al. 2003), and fast sausage waves (Su et al. 2012b; Tian et al. 2016). A remarkable application, namely MHD coronal seismology, derived from those observational parameters is to diagnose the properties of coronal plasma along the loops as well as the magnetic field strength (e.g., Nakariakov \& Verwichte 2005; De Moortel \& Nakariakov 2012; Yuan \& Van Doorsselaere 2016a). This can also greatly improve our current understanding of coronal heating and magnetic reconnection theory (Nakariakov et al. 1999; Goossens et al. 2013).

Most recent studies of loop oscillations only focus on the imaging observations (e.g., Anfinogentov et al. 
2015; Zimovets \& Nakariakov 2015; Goddard \& Nakariakov 2016) and here we utilize the spectroscopic data to measure the Doppler shift oscillations on the Fe XXI $1354.09 \AA$ line which is formed at the temperature of $\sim 11$ MK. Our results provide strong evidences for fast kink oscillations in a flare loop. The observations are taken from the joint observations, including the Interface Region Imaging Spectrograph (IRIS, De Pontieu et al. 2014), the Atmospheric Imaging Assembly (AIA, Lemen et al. 2012) and Helioseismic and Magnetic Imager (HMI, Schou et al. 2012) on board $S D O$, the X-ray Telescope (XRT, Golub et al. 2007) aboard Hinode, the Nobeyama Radioheliograph (NoRH, Hanaoka et al. 1994), and the Geostationary Operational Environment Satellites (GOES, Aschwanden 1994).

\section{Observations and Data Analysis}

The M7.1 flare under our study occurs in AR 12192 on 2014 October 27, and its derived position is S12W42. It starts at about 00:06 UT, peaks nearly at 00:34 UT, and stops at around 00:44 UT. Figure 1] shows the simultaneous snapshots with the same field-of-view (FOV) of $120^{\prime \prime} \times 128^{\prime \prime}$ at SXR, EUV/UV and magnetogram observed by Hinode/XRT ( $1^{\prime \prime} /$ pixel $), S D O / A I A\left(~ 0.6^{\prime \prime} /\right.$ pixel $)$, IRIS/SJI $\left(\sim 0.33^{\prime \prime} / \mathrm{pixel}\right)$, and SDO/HMI $\left(\sim 0.6^{\prime \prime} / \mathrm{pixel}\right)$, respectively. The XRT, AIA, and HMI images have been pre-processed with the routines of 'xrt_prep.pro' (Golub et al. 2007), 'aia_prep.pro' (Lemen et al. 2012), and 'hmi_prep.pro' (Schou et al. 2012) in the solar software (SSW) package, respectively. First, to coalign the XRT and AIA imaging observations, we use Be_med filter and 193 $\AA$ images in which a hot flare loop (indicated by the black contours) is clearly visible and can be identified easily. Second, the AIA $1600 \AA$ image is applied to co-align with the SJI $1330 \AA$ image (Li et al. 2014; Cheng et al. 2015), because they both contain the continuum emission from the temperature minimum (marked by the purple contours). A broad and bright loop is visible in the XRT Be_med filter, AIA $193 \AA$ and $131 \AA$ images, and the legs of loop are rooted individually in two polarities (see the blue and red contours). The loop structure is weaker in other AIA channels, such as the tip (or cusp-like tip) of the loop that is hardly seen in AIA $94 \AA$ and invisible in AIA $211 \AA$ and $171 \AA$. The flare ribbons are best shown in AIA $1600 \AA$ and SJI $1330 \AA$. These observations suggest that the loop structure, especially the loop top, is very hot, which can be greater than 11 MK.

In order to investigate the loop oscillations carefully, we select an area on the loop-top region that is crossed by the IRIS slit. It is bounded by two short green lines in Figure 1, In this observation, the flare is observed in a 'sit-and-stare' mode with a step cadence of $\sim 16.2 \mathrm{~s}$, which provides an opportunity to study the evolution of flare spectra. First, two routines in the SSW package, namely 'iris_orbitval_corr_12.pro' (Tian et al. 2014; Cheng et al. 2015) and 'iris_prep_despike.pro' (De Pontieu et al. 2014) are applied to the observed spectra. And here a relatively strong neutral

line (i.e., O I $1355.60 \AA$ ) are used to process the absolute wavelength calibration (see., De Pontieu et al. 2014; Tian et al. 2015; Li et al. 2017b). The multi-Gaussian functions superimposed on a linear background are applied to fit the observed spectra. In the final step, the hot line of Fe XXI $1354.09 \AA$ 
can be extracted from the fitting result. The detailed description of the fitting method can be seen in our previous papers (Li et al. 2015b, 2016a).

Figure 2 shows the flare spectra and the fitting results at six selected time. It is well known that Fe XXI $1354.09 \AA$ is a broad and hot $(\sim 11 \mathrm{MK})$ line, whereas it is always blended with other narrow and cool emission lines, such as C I 1354.29 $\AA$, Fe II 1353.02, 1354.01 and $1354.76 \AA$, Si II 1352.63 and $1353.72 \AA$, and some unidentified emission lines (Doschek et al. 1975; Mason et al. 1986; Innes et al. 2003; Li et al. 2015c; Young et al. 2015). Here, the flare spectrum (black) is taken from the loop-top position indicated by a short purple line. The purple overlaid profile represents the multi-Gaussian fitting result, and the orange horizontal line is a linear background. The overlaid turquoise profile is the Fe XXI $1354.09 \AA$ line extracted from the fitting result. The other fitting parameter such as the line center is also overplotted in each subfigure (vertical turquoise line) and used to calculate the Doppler velocity (Li et al. 2015b).

\section{Results}

\subsection{Doppler shift oscillations}

Figure 3 shows the space-time images of Doppler velocity (upper) and line-integrated intensity (lower) at Fe Xxi $1354.09 \AA$. The selected loop-top area is bounded by two green lines, and the plus signs ('+') mark the time of the flare spectra in Figure 2, A pronounced Doppler shift oscillation is observed in the loop-top area from about 00:20 UT to 00:25 UT. It begins from a red-shifted velocity, and then changes to a blue-shifted velocity, such oscillatory behavior repeats at least two times, as indicated by the solid arrows in the upper panel. In other words, the oscillatory behaviors last for at least two cycles if we define one cycle as a pair of red and blue shifts. After two visible changes from red to blue shifts, the Doppler shifts become very weak. However, no such obvious oscillations can be seen in the space-time image of line-integrated intensity during the same time intervals, as seen in the lower panel.

The upper panel in Figure 4 shows the time series of Doppler shift (black) and line-integrated intensity (turquoise), they are averaged over the IRIS slit positions between two green lines in Figure 3, As a comparison, the SXR light curves at 1.0-8.0 $\AA$ (black) and 0.5-4.0 $\AA$ (turquoise) from GOES and normalized microwave fluxes at $17 \mathrm{GHz}$ (solid purple) and $34 \mathrm{GHz}$ (dash purple) from the NoRH are also given in the lower panel of Figure 4, The time series of Doppler shift exhibit six pronounced and regular peaks between around 00:10 UT-00:25 UT, which are labeled by the number ticks. The oscillations during the peaks ' 5 ' and ' 6 ' exhibit the changing from red to blue wings, as marked by the solid arrows. The same oscillations can be observed during the peaks ' 1 ' and ' 2 ', which also display a change from red to blue wings. Hoverer, there are only red-shifted oscillations during the peaks ' 3 ' and ' 4 ', and they are always dominated by red wings. It is also noted that the onset of these Doppler shift oscillations occurs in the impulsive phase of this flare. On the other hand, none of the similar oscillatory signature is revealed in the line-integrated intensity 
of Fe XXI 1354.09 A, normalized microwave fluxes and SXR light curves. But the enhancement of line-integrated intensity is simultaneous with the appearance of Doppler shift oscillations, e.g., in peaks ' 1 ', and ' 5 '. In peak ' 4 ', the line-integrated intensity decreases when the Doppler shift tends to be red-shifted. It is well known that the microwave emissions are produced by the nonthermal electron beams trapped in a flare loop during the impulsive phase of solar flare (see., Kundu et al. 1994; White et al. 2003; Reznikova \& Shibasaki 2011; Dolla et al. 2012; Asai et al. 2013; Kumar et al. 2016). Although our observations does not show in-phase oscillations in the normalized microwave fluxes at $17 \mathrm{GHz}$ and $34 \mathrm{GHz}$, it is interesting to note that the pulse peaks of microwave emissions proceed the onset of Doppler shift oscillations by $\sim 1$ minute, indicating the non-thermal electrons produced by magnetic reconnection before Doppler shift oscillations in this flare.

To obtain the oscillatory period, a wavelet analysis method is used (Torrence \& Compo 1998; Yuan et al. 2011; Tian et al. 2012; Li \& Zhang 2017). Firstly, a 5-min running average in the time series of Doppler velocity is considered as a background emission, as indicated by the green profile in the upper panel of Figure 4. Secondly, the detrended time series of Doppler velocity are calculated from the original time series by removing the background emission (5-min running average). Figure 5 (a) shows the detrended velocity, and they exhibit six distinct peaks, which are well matched with those peaks in the upper panel of Figure 4, as labeled by the number ticks. Figure 5 (b) and (c) display wavelet power spectrum and global wavelet, respectively. The oscillatory period can be measured from the peak value of the global wavelet power, and the uncertainty is thought to be the half width at half-maximum value (Tian et al. 2016). Finally, a dominant period with an error bar can be estimated for the detrended Doppler velocity of Fe XXI $1354.09 \AA$, which is about $3.1 \pm 0.6$ minutes. The wavelet power spectrum shows that these Doppler shift oscillations are from $~ 00: 10$ UT to $\sim 00: 25$ UT, which correspond to the time interval during the six peaks in the time series of Doppler velocity (see Figure 4).

\subsection{Flare loop geometry}

Thanks to the high spatial resolution of imaging observations, we are able to study the morphological structure of the oscillatory positions. Due to data gaps in XRT observations, only a few XRT images are presented to show the flare loop structure at high temperature during the time interval of the pronounced oscillations. The SXR and EUV images are carefully selected to avoid data saturation (see also., Li et al. 2015a; Ning 2017) and plotted with the Y-axis along the direction of IRIS slit, e.g., 45 degree rotation. Figure 6 displays the loop structure and its movement from peak ' 5 ' to peak ' 6 ' at XRT Be_med filter and AIA $193 \AA$, it also shows the line-of-sight (LOS) magnetogram with the same FOV. Figure 6 shows that double footpoints which connected by this flare loop root in the positive and negative magnetic fields, respectively. We trace this flare loop in XRT images and overlaid on each AIA $193 \AA$ image (purple line). The region of interested is the loop-top area which is bounded by two green lines here. The bright loop in AIA $193 \AA$ images 
is cospatial with the overlaid SXR loop. It is noted that the bright loop-top region (outlined with two green lines) is crossed by the slit of IRIS (turquoise line).

Further, we also calculate some parameters of this flare loop. The apparent loop length is estimated to be $\sim 54 \mathrm{Mm}$ according to the XRT images in Figure 6, as shown by the purple line. Then we can obtain the deprojected loop length $(\mathrm{L} \approx 81 \mathrm{Mm})$ when considering the projection effect

due to the derived position of solar flare (Aschwanden et al. 2002; Kumar et al. 2013; Li 2017). The loop width $(w)$ is also calculated from the XRT images. We first average the intensities between two green lines and obtain the flux along the X-direction. Then we fit the flux with a Gaussian function. Finally, the full width at half maximum (FWHM) of Gaussian function is considered as the loop width, which is about $9.8 \mathrm{Mm}$. Thus, the geometric ratio between loop width and length $(w / \mathrm{L})$ is around 0.12 . This geometric ratio is quite reasonable, given $w=2 \times r$ ( $\mathrm{r}$ is the radius of flare loop), therefore, the aspect ratio between loop radius and length $(r / \mathrm{L})$ is about 0.06 , which is consistent with previous findings in the coronal loops (Aschwanden et al. 2002).

\subsection{Temperature and density}

To investigate the temperature structure of this flare loop, we perform the differential emission measure (DEM) analysis using AIA data at six EUV wavelengths (see., Cheng et al. 2012; Sun et al. 2014; Shen et al. 2015). Figure 7 (a)-(e) shows the DEM profiles in the loop-top region (marked by the red box of pi in Figure 66) with a FOV of $2.4^{\prime \prime} \times 2.4^{\prime \prime}$ at the selected time. While Figure 7 (f) gives the DEM profiles outside flare loop, as indicated by the red box of po. The average temperature $(T)$ and emission measure (EM) at each time are estimated within a confident temperature $(\log T)$ range of 5.5-7.5 (Ning et al. 2016). The best-fitted DEM solution of the observation is indicated by the black profile. Then the Monte Carlo (MC) realizations of the observations are applied to estimate the DEM uncertainty, as indicated by the top and bottom color rectangles (see., Cheng et al. 2012; Li et al. 2016b, d, d). It gives much lower value of the uncertainty on our DEM analysis, especially at the high temperature regions, i.e., panels (c) and (d).

The upper panel of Figure 8 is the time series of the Doppler shift and temperature at the flare loop-top region, and the lower panel shows the EM variation and EUV fluxes at AIA $193 \AA$ $(\sim 20 \mathrm{MK}), 131 \AA(\sim 11 \mathrm{MK}), 94 \AA(\sim 6.3 \mathrm{MK}), 211 \AA(\sim 2.0 \mathrm{MK})$, and $171 \AA(\sim 0.63 \mathrm{MK})$ (Lemen et al. 2012). The temperature rises rapidly at the onset of Doppler shift oscillations (e.g., peak ' 1 ' \& ' 5 '), which is almost in phase with the enhancement of the EUV fluxes at AIA $131 \AA$ and $193 \AA$ passbands $(\geq 11 \mathrm{MK})$. The temperature increases here might be due to hot emission from the loop top that are clearly seen in the XRT Be_med and AIA $193 \AA$ images (Figures $\measuredangle$ ) and also crossed by the slit of IRIS. However, the EUV fluxes in other channels lags by several minutes and such in-phase correspondence can not be found, implying that the Doppler shift oscillations only take place in the hot loop, i.e., at least 11 MK. The EM varies very slowly throughout the entire impulsive phase. It keeps nearly constant during the occurrence of Doppler shift oscillations, suggesting the incompressible flare loop. The average EM in the flare loop-top area between 
$\sim 00: 20$ UT and $\sim 00: 25$ UT is estimated to be $\sim 3 \times 10^{30} \mathrm{~cm}^{-5}$. We further estimate the average number density from equation 1 (see also., Zhang \& Ji 2014; Su et al. 2015; Ning et al. 2016; Zhang et al. 2016b), which is about $5.5 \times 10^{10} \mathrm{~cm}^{-3}$. This value agrees well with previous findings in flare loops (Aschwanden \& Benz 1997), for example, $n_{e} \approx(2-25) \times 10^{10} \mathrm{~cm}^{-3}$ in SXR loops.

$$
n_{e}=\sqrt{\frac{E M}{w}}
$$

\section{Discussions}

\subsection{Error analysis}

We notice that the Doppler velocities shown in Figure 4 are not pronounced. For example, they are no more than $\pm 25 \mathrm{~km} \mathrm{~s}^{-1}$ during the Doppler shift oscillations. The Doppler velocity in this study is calculated from the subtraction between the fitting line center and the rest wavelength, and there could be two quantities producing the errors. One is the fitting line center derived from the multi-Gaussian fitting method. The multi-Gaussian fitting procedure (detail see., Li et al. 2015b, 2016a) used to obtain the Fe Xxi $1354.09 \AA$ here has included as much as possible the known/unknown blending emission lines in this dataset. The final fit as well as the extracted Fe XXI $1354.09 \AA$ line profile (see Figure 2) appear to be successful at the selected positions. Thus the derived fitting line center is convincing. The other one is the rest wavelength. The formation temperature of Fe Xxi $1354.09 \AA$ is $\sim 11 \mathrm{MK}$, which is too hot to be observed in the quiet-Sun spectrum. In this paper, we set the rest wavelength as $1354.09 \AA$, which is the average value from

the recent IRIS spectral observations, i.e., $1354.08 \AA-1354.1 \AA$ (e.g., Polito et al. 2015; Tian et al. 2015; Sadykov et al. 2015; Brosius et al. 2016). And the uncertainty is around $\pm 0.01 \AA$, which corresponds to the Doppler shift of about $\pm 2.2 \mathrm{~km} \mathrm{~s}^{-1}$. This uncertainty on rest wavelength of Fe XXI $1354.09 \AA$ might change the Doppler shift slightly but does not have a significant impact on our results. On the other hand, Tian et al. (2016) find that the Doppler velocity of Fe Xxi $1354.09 \AA$ in a flare loop is much smaller than that in the flare ribbons, which is similar as our results.

\subsection{MHD waves}

The oscillations in flare/coronal loops are usually related to MHD processes Roberts 2000; De Moortel \& Nakariakov 2012; Tian et al. 2016), such as slow magnetoacoustic waves, fast sausage waves and fast kink waves. In this paper, the loop oscillations are observed in Doppler shift of Fe XXI $1354.09 \AA$, but there are no apparent corresponding intensity fluctuations in line-integrated intensity of Fe XXI $1354.09 \AA$ (Figure 4) or AIA EUV fluxes (Figure 8). In DEM analysis, the EM is also relatively stable during the Doppler shift oscillations. These facts suggest that the flare loop oscillates in an incompressible mode, and further rule out the possibility of slow magnetoacoustic 
waves because they are compressible magnetoacoustic waves (Roberts 2000; Wang et al. 2002, 2003; Aschwanden 2005). The loop oscillations are unlikely to be interpreted as fast sausage waves either. The sausage oscillations within short periods $(<60 \mathrm{~s})$ are often decayless or weakly damping when the cutoff condition and those within long periods $(>60 \mathrm{~s})$ are substantially compressible and transverse (Gruszecki et al. 2012; Vasheghani Farahani et al. 2014; Tian et al. 2016). Moreover, they could produce density variation, which can easily modulate the periodic oscillations in microwave emission Rosenberg 1970; Aschwanden 2005; Antolin \& Van Doorsselaere 2013; Reznikova et al. 2015). The loop oscillations here are incompressible, damping, and no corresponding intensity fluctuations are detected in EUV and microwave emissions. All those theoretical predictions of fast sausage waves argue against our observations.

The observed loop oscillations are most probably fast kink waves. The oscillations produced by kink waves can produce the intensity and Doppler shift based on the column depth from different viewing angles. If the spectral slit is aligned along the loop, only Doppler shift oscillations are expected from the LOS direction (Yuan \& Van Doorsselaere 2016a). In addition, the kink oscillations can also decay rapidly (Nakariakov et al. 1999; Aschwanden 2005; Zimovets \& Nakariakov 2015; Yuan et al. 2016). All these theories are consistent with our observations. In this event, a flare loop is detected to be corresponded to the Doppler shift oscillations. The observed oscillatory period in this flare loop is $\sim 3.1 \pm 0.6$ minutes. That is to say, the dominant period of kink oscillations is $\sim 3.1$ minutes. On the other hand, according to the period $\left(\mathrm{P}_{k}\right)$ of the standing kink oscillations, we can diagnose the magnetic field strength $(B)$ in this flare loop from equation 2 (Roberts et al. 1984; Nakariakov \& Ofman 2001; Wang et al. 2002; Aschwanden 2005; Tian et al. 2012).

$$
B \approx \frac{v_{A}}{2.18 \times 10^{11}} n_{e}^{\frac{1}{2}}, \quad v_{A}=v_{k}\left(\frac{2}{1+n_{0} / n_{e}}\right)^{-\frac{1}{2}}, \quad v_{k}=\frac{2 L}{P_{k}} .
$$

Here, $v_{A}$ is the local Alfvén speed, $v_{k}$ is the phase speed of the standing kink wave in its fundamental mode, $n_{e}$ and $n_{0}$ are the electron densities inside and outside flare loop, respectively. As mentioned in section 3, the deprojected length (L) of this flare loop (Figure 6) is $\sim 81 \mathrm{Mm}$, thus the kink speed can be estimated to be $\sim 870 \mathrm{~km} \mathrm{~s}^{-1}$. Meanwhile, the number density $\left(n_{e}\right)$ inside flare loop can be estimated from equation 1. However, it is impossible to find a loop width $(w)$ outside flare loop. Therefore, the effective LOS depth $(l \sim \sqrt{H \pi r})$ is used to estimate the number density outside flare loop region (po), which is $\sim 4 \times 10^{10} \mathrm{~cm}$ (Zucca et al. 2014; Su et al. 2016). Then we can obtain the density ratio $\left(n_{0} / n_{e} \sim 0.03\right)$ between outside (po) and inside (pi) flare loop at about 00:24:13 UT, as seen in Figure 7(d) and (f). The Alfvén speed can be estimated to be about $630 \mathrm{~km} \mathrm{~s}^{-1}$, and the magnetic filed strength in this flare loop can be estimated to be $\sim 68 \mathrm{G}$, which agrees with pervious results, i.e., 60-120 G in flare loop (see Qiu et al. 2009). In our observations, the density ratio $\left(n_{0} / n_{e}\right)$ around flare loop is less than the typical density ratio (i.e., $\left.0.1-0.5\right)$ around coronal loop (Aschwanden 2005), suggesting that the number density in flare loop is higher than that in coronal loop. 


\subsection{Fourier analysis}

The upper panel in Figure 4 exhibits that there are at least two oscillatory periods in the time series of Doppler shift of Fe Xxi $1354.09 \AA$. One is the shorter period ( $\sim 3.1$ minutes) which has been obtained in section 3.1. The other one seems to be a longer period, and the time series of line-integrated intensity also display the similar longer period. To extract the longer period, we perform a Fourier analysis using the method described by Yuan et al. (2011), which is based on the Lomb-Scargle periodogram technique (Scargle 1982; Horne \& Baliunas 1986).

Figure 9 shows the results of spectral analysis. The Fourier analysis is applied to the detrended time series after removing the background emission, which is the 12-min running average of the time series (see., Tian et al. 2012; Li \& Zhang 2017). Here, a different averaging time window only results in changing in relative power of the peaks (Yuan et al. 2011). Panel (a) gives the normalized power spectrum of detrended Doppler shift. From which, we can extract at least two components of periods, $\mathrm{P}_{1}$ and $\mathrm{P}_{2}$. We also calculate the error bars as the uncertainties of periods, which are the half width at half-maximum value of the Fourier power (Yuan et al. 2011; Tian et al. 2016). The shorter dominant period $\left(\mathrm{P}_{1}\right)$ within an error bar is around $3.1 \pm 0.6$ minutes. The dominant period of $\mathrm{P}_{1}$ agrees well with the wavelet analysis result (Figure 5). While the longer dominant period $\left(\mathrm{P}_{2}\right)$ within an error bar is about $10 \pm 4$ minutes. This longer period can also be detected from the dtrended time series of line-integrated intensity of Fe XXI $1354.09 \AA$ (b). To illustrate this longer period, we also perform Fourier analysis of the detrended fluxes from GOES 1.0-8.0 (c), 0.5-4.0 $\AA$ (d) in SXR passbands, and AIA $193 \AA$ (f), $131 \AA$ (f) in EUV wavelengths. The normalized power spectra show that all these detrended fluxes display a longer dominant period of $\sim 10$ minutes, which is same as that in Doppler shift of Fe Xxi 1354.09 $\AA$. On the other hand, the shorter period can not be detected in these detrended fluxes, which further confirms that the 3.1-min oscillations can only be observed in Doppler shift of Fe Xxi $1354.09 \AA$.

As mentioned above, the Doppler shift oscillations within a period of $\sim 3.1$ minutes $\left(\mathrm{P}_{1}\right)$ can be interpreted as the standing kink oscillations in the hot flare loop. On the other hand, the longer period $\left(\mathrm{P}_{2}\right)$ is different from the shorter one $\left(\mathrm{P}_{1}\right)$, because it can be observed simultaneously in SXR and EUV fluxes, as well as in the time series of Doppler shift and line-integrated intensity of Fe XXI $1354.09 \AA$. Those facts imply the process of periodic energy release in this flare (Tian et al. 2015; Li et al. 2017a). The longer period is most likely to be linked to the quasi-periodic pulsations (QPPS) during the solar flare, which seems to be caused by a MHD wave in slow mode (Fang et al. 2015; Van Doorsselaere et al. 2016). Based on the formation temperature $(T \sim 11 \mathrm{MK})$ of Fe XXI $1354.09 \AA$, the local sound speed $\left(v_{s} \approx 152 \sqrt{T / \mathbf{M K}}\right)$ in flare loop is estimated to be $\sim 500 \mathrm{~km} \mathrm{~s}^{-1}$ (e.g., Nakariakov \& Ofman 2001; Kumar et al. 2013, 2015). While the deprojected length (L) and longer period $\left(\mathrm{P}_{2}\right)$ of this flare loop have been identified as $\sim 81 \mathrm{Mm}$ and $\sim 10 \pm 4$ minutes, then the phase speed of this flare loop $\left(v_{h}=2 \mathrm{~L} / \mathrm{P}_{2}\right)$ can be estimated as about $200-450 \mathrm{~km} \mathrm{~s}^{-1}$. This phase speed is slower than the local sound speed, implying it might be a slow MHD wave (Mandal et al. 2016). In other words, the longer period $\left(\mathrm{P}_{2}\right)$ might be explained as the MHD wave in a slow mode. 
To compare the Doppler shift oscillations, we also use the sine function with a damping amplitude (equation [3) to fit the time series of Doppler shift during peaks ' 5 ' and '6' (e.g., Nakariakov et al. 1999; Wang et al. 2002; Anfinogentov et al. 2015), because the Doppler shift oscillations are most pronounced during this time interval. Figure 8 (upper) shows the fitting result, the overlaid turquoise profile gives the best fitting, and it appears to match well with the Doppler velocities (black).

$$
v(t)=v_{0}+v_{m} \sin \left(\frac{2 \pi}{P_{1}} t+\psi\right) e^{-\frac{t}{\tau}} .
$$

Here, $v_{0}$ is the initial Doppler velocity, $v_{m}$ is the initial oscillatory amplitude, and $\mathrm{P}_{1}, \psi$, and $\tau$ are the shorter dominant period, initial phase, and decay time of the oscillations, respectively.

\section{Conclusions}

Using observations from multi-instruments, i.e., IRIS, SDO/AIA, SDO/HMI, Hinode/XRT and $\mathrm{NoRH}$, we investigate the Doppler shift oscillations in a hot loop during a GOES M7.1 flare on 2014 October 27. The main results are summarized as follows.

(I) The Doppler shift oscillations are detected in a hot flare line, i.e., Fe xxi $1354.09 \AA$ from IRIS spectroscopic observations. The time series of Doppler shift start the oscillations from red to blue wings (peaks ' 1 ' \& ' 2 '), and then oscillate at red wings (peaks ' 3 ' \& ' 4 '), finally end the oscillations from red to blue wings (peaks ' 5 ' \& ' 6 ').

(II) Based on the wavelet analysis, a dominant period of $\sim 3.1$ minutes is obtained from the Doppler shift oscillations. The Fourier analysis and sine function fitting provide an additional evidence of this shorter period in the Doppler shift of Fe XXI $1354.09 \AA$.

(III) The Doppler shift oscillations at the shorter period are from the flare loop-top region. The temperature of the corresponding flare loop is not less than $11 \mathrm{MK}$, which can be confirmed in the XRT Be_med filter, AIA 193 and $131 \AA$ images and DEM analysis in section 3.3.

(IV) There are no apparent corresponding oscillations within the period of $\sim 3.1$ minutes in the line-integrated intensity of Fe XXI 1354.09 A, AIA EUV fluxes, or microwave emissions, suggesting that the hot flare loop is incompressible. This is consistent with the DEM analysis, which shows slightly change on EMs during the Doppler shift oscillations.

(V) The 3.1-min period of Doppler shift oscillations at Fe XXI $1354.09 \AA$ might be explained by the MHD wave in a standing kink mode. Thus, the magnetic filed strength in this flare loop is estimated to be $\sim 68 \mathrm{G}$, and the density ratio between outside and inside flare loop is $\sim 0.03$.

(VI) The 10-min period oscillations can be observed in GOES SXR fluxes, SDO/AIA EUV light curves and the time series of Doppler shift and line-integrated intensity, implying the periodic energy release in this flare, and it might be caused by a slow mode MHD wave. 
The authors would like to thank the anonymous referee for his/her valuable comments. We also acknowledge Prof. H. Tian, L. P. Li, D. Yuan, P. F. Chen, X. Cheng and Y. Guo for their inspiring discussions. We appreciate the teams of IRIS, GOES, NoRH, Hinode/XRT, SDO/AIA and $S D O / \mathrm{HMI}$ for their open data use policy. This study is supported by NSFC under grants 11603077, 11573072, 11773079, 11773061, 11473071, 11333009, XDA15052200, KLSA201708, 973 program (2014CB744200), and Laboratory No. 2010DP173032. This work is also supported by the Youth Fund of Jiangsu Nos. BK20161095, BK20171108, and BK20141043, Dr. Q. M. Zhang is supported by the Surface Project of Jiangsu No. BK 20161618 and the Youth Innovation Promotion Association CAS, and Dr. Y. N, Su is also supported by one hundred talent program of Chinese Academy of Sciences. The authors wish to thank the International Space Science Institute in Beijing (ISSI-BJ) for supporting and hosting the meeting of the International Team on "Magnetohydrodynamic Seismology of the Solar Corona in the Era of SDO/AIA", during which the discussions leading to this publication were held.

\section{REFERENCES}

Anfinogentov, S. A., Nakariakov, V. M., \& Nisticò, G. 2015, A\&A, 583, A136

Antolin, P., \& Van Doorsselaere, T. 2013, A\&A, 555, A74

Asai, A., Kiyohara, J., Takasaki, H., et al. 2013, ApJ, 763, 87

Aschwanden, M. J. 1994, Sol. Phys., 152, 53

Aschwanden, M. J., \& Benz, A. O. 1997, ApJ, 480, 825

Aschwanden, M. J., Fletcher, L., Schrijver, C. J., \& Alexander, D. 1999, ApJ, 520, 880

Aschwanden, M. J., de Pontieu, B., Schrijver, C. J., \& Title, A. M. 2002, Sol. Phys., 206, 99

Aschwanden, M. J. 2005, Pour la Science,

Brosius, J. W., Daw, A. N., \& Inglis, A. R. 2016, ApJ, 830, 101

Cheng, X., Zhang, J., Saar, S. H., \& Ding, M. D. 2012, ApJ, 761, 62

Cheng, X., Ding, M. D., \& Fang, C. 2015, ApJ, 804, 82

De Moortel, I., Ireland, J., Hood, A. W., \& Walsh, R. W. 2002, A\&A, 387, L13

De Moortel, I., \& Nakariakov, V. M. 2012, Philosophical Transactions of the Royal Society of London Series A, 370, 3193

De Pontieu, B., Title, A. M., Lemen, J. R., et al. 2014, Sol. Phys., 289, 2733

Dolla, L., Marqué, C., Seaton, D. B., et al. 2012, ApJ, 749, L16 
Doschek, G. A., Dere, K. P., Sandlin, G. D., et al. 1975, ApJ, 196, L83

Fang, X., Yuan, D., Van Doorsselaere, T., Keppens, R., \& Xia, C. 2015, ApJ, 813, 33

Golub, L., Deluca, E., Austin, G., et al. 2007, Sol. Phys., 243, 63

Goddard, C. R., \& Nakariakov, V. M. 2016, A\&A, 590, L5

Goossens, M., Van Doorsselaere, T., Soler, R., \& Verth, G. 2013, ApJ, 768, 191

Gruszecki, M., Nakariakov, V. M., \& Van Doorsselaere, T. 2012, A\&A, 543, A12

Hanaoka, Y., Shibasaki, K., Nishio, M., et al. 1994, Proceedings of Kofu Symposium, 35

Horne, J. H., \& Baliunas, S. L. 1986, ApJ, 302, 757

Innes, D. E., McKenzie, D. E., \& Wang, T. 2003, Sol. Phys., 217, 267

Inglis, A. R., \& Nakariakov, V. M. 2009, A\&A, 493, 259

Kliem, B., Dammasch, I. E., Curdt, W., \& Wilhelm, K. 2002, ApJ, 568, L61

Kumar, P., Innes, D. E., \& Inhester, B. 2013, ApJ, 779, L7

Kumar, P., Nakariakov, V. M., \& Cho, K.-S. 2015, ApJ, 804, 4

Kumar, P., Nakariakov, V. M., \& Cho, K.-S. 2016, ApJ, 822, 7

Kundu, M. R., White, S. M., Gopalswamy, N., \& Lim, J. 1994, ApJS, 90, 599

Lemen, J. R., \& Title, A. M., \& Akin, D. J., et al. 2012, Sol. Phys., 275, 17

Lipa, B. 1978, Sol. Phys., 57, 191

Li, D., Ning, Z. J., \& Zhang, Q. M. 2015a, ApJ, 807, 72

Li, D., Ning, Z. J., \& Zhang, Q. M. 2015b, ApJ, 813, 59

Li, D., Innes, D. E., \& Ning, Z. J. 2016a, A\&A, 587, A11

Li, D., Ning, Z., \& Su, Y. 2016b, Ap\&SS, 361, 301

Li, D., Zhang, Q. M., Huang, Y., Ning, Z. J., \& Su, Y. N. 2017a, A\&A, 597, L4

Li, D., Ning, Z. J., Huang, Y., \& Zhang, Q. M. 2017b, ApJ, 841, L9

Li, D., \& Zhang, Q. M. 2017, MNRAS, 471, L6

Li, D. 2017, Research in Astronomy and Astrophysics, 17, 040

Li, L. P., Peter, H., Chen, F., \& Zhang, J. 2014, A\&A, 570, A93 
Li, L. P., Zhang, J., Su, J. T., \& Liu, Y. 2016c, ApJ, 829, L33

Li, L., Zhang, J., Peter, H., et al. 2016d, Nature Physics, 12, 847

Li, T., \& Zhang, J. 2015, ApJ, 804, L8

Li, Y., Ding, M. D., Qiu, J., \& Cheng, J. X. 2015c, ApJ, 811, 7

Li, Y. P., \& Gan, W. Q. 2008, Sol. Phys., 247, 77

Mariska, J. T. 2005, ApJ, 620, L67

Mariska, J. T. 2006, ApJ, 639, 484

Mandal, S., Yuan, D., Fang, X., et al. 2016, ApJ, 828, 72

Mason, H. E., Shine, R. A., Gurman, J. B., \& Harrison, R. A. 1986, ApJ, 309, 435

Melnikov, V. F., Reznikova, V. E., Shibasaki, K., \& Nakariakov, V. M. 2005, A\&A, 439, 727

Nakariakov, V. M., Ofman, L., Deluca, E. E., Roberts, B., \& Davila, J. M. 1999, Science, 285, 862

Nakariakov, V. M., \& Ofman, L. 2001, A\&A, 372, L53

Nakariakov, V. M., \& Verwichte, E. 2005, Living Reviews in Solar Physics, 2, 3

Nakariakov, V. M., \& Melnikov, V. F. 2009, Space Sci. Rev., 149, 119

Ning, Z. 2014, Sol. Phys., 289, 1239

Ning, Z., Li, D., \& Zhang, Q. M. 2016, Sol. Phys., 291, 1783

Ning, Z. 2017, Sol. Phys., 292, 11

Nisticò, G., Nakariakov, V. M., \& Verwichte, E. 2013, A\&A, 552, A57

Ofman, L., \& Wang, T. 2002, ApJ, 580, L85

Polito, V., Reeves, K. K., Del Zanna, G., Golub, L., \& Mason, H. E. 2015, ApJ, 803, 84

Qiu, J., Gary, D. E., \& Fleishman, G. D. 2009, Sol. Phys., 255, 107

Reznikova, V. E., \& Shibasaki, K. 2011, A\&A, 525, A112

Reznikova, V. E., Van Doorsselaere, T., \& Kuznetsov, A. A. 2015, A\&A, 575, A47

Roberts, B., Edwin, P. M., \& Benz, A. O. 1984, ApJ, 279, 857

Roberts, B. 2000, Sol. Phys., 193, 139

Rosenberg, H. 1970, A\&A, 9, 159 
Sadykov, V. M., Vargas Dominguez, S., Kosovichev, A. G., et al. 2015, ApJ, 805, 167

Scargle, J. D. 1982, ApJ, 263, 835

Schou, J., Scherrer, P. H., Bush, R. I., et al. 2012, Sol. Phys., 275, 229

Schrijver, C. J., Aschwanden, M. J., \& Title, A. M. 2002, Sol. Phys., 206, 69

Shen, Y., \& Liu, Y. 2012, ApJ, 753, 53

Shen, Y.-D., Liu, Y., Su, J.-T., et al. 2013, Sol. Phys., 288, 585

Shen, Y., Liu, Y., Liu, Y. D., et al. 2015, ApJ, 814, L17

Su, J. T., Shen, Y. D., \& Liu, Y. 2012a, ApJ, 754, 43

Su, J. T., Shen, Y. D., Liu, Y., Liu, Y., \& Mao, X. J. 2012b, ApJ, 755, 113

Su, W., Cheng, X., Ding, M. D., Chen, P. F., \& Sun, J. Q. 2015, ApJ, 804, 88

Su, W., Cheng, X., Ding, M. D., et al. 2016, ApJ, 830, 70

Sun, J. Q., Cheng, X., \& Ding, M. D. 2014, ApJ, 786, 73

Tan, B., \& Tan, C. 2012, ApJ, 749, 28

Tian, H., McIntosh, S. W., \& De Pontieu, B. 2011, ApJ, 727, L37

Tian, H., McIntosh, S. W., Wang, T., et al. 2012, ApJ, 759, 144

Tian, H., DeLuca, E., Reeves, K. K., et al. 2014, ApJ, 786, 137

Tian, H., Young, P. R., Reeves, K. K., et al. 2015, ApJ, 811, 139

Tian, H., Young, P. R., Reeves, K. K., et al. 2016, ApJ, 823, L16

Torrence, C., \& Compo, G. P. 1998, Bulletin of the American Meteorological Society, 79, 61

Van Doorsselaere, T., Kupriyanova, E. G., \& Yuan, D. 2016, Sol. Phys., 291, 3143

Vasheghani Farahani, S., Hornsey, C., Van Doorsselaere, T., \& Goossens, M. 2014, ApJ, 781, 92

Wang, T., Solanki, S. K., Curdt, W., Innes, D. E., \& Dammasch, I. E. 2002, ApJ, 574, L101

Wang, T. J., Solanki, S. K., Innes, D. E., Curdt, W., \& Marsch, E. 2003, A\&A, 402, L17

Wang, T., Ofman, L., Davila, J. M., \& Su, Y. 2012, ApJ, 751, L27

White, S. M., Krucker, S., Shibasaki, K., et al. 2003, ApJ, 595, L111

Yang, S., \& Xiang, Y. 2016, ApJ, 819, L24 
Young, P. R., Tian, H., \& Jaeggli, S. 2015, ApJ, 799, 218

Yuan, D., Nakariakov, V. M., Chorley, N., \& Foullon, C. 2011, A\&A, 533, A116

Yuan, D., \& Van Doorsselaere, T. 2016a, ApJS, 223, 23

Yuan, D., \& Van Doorsselaere, T. 2016b, ApJS, 223, 24

Yuan, D., Su, J., Jiao, F., \& Walsh, R. W. 2016, ApJS, 224, 30

Zhang, Q. M., \& Ji, H. S. 2014, A\&A, 567, A11

Zhang, Q. M., Li, D., \& Ning, Z. J. 2016a, ApJ, 832, 65

Zhang, Q. M., Ji, H. S., \& Su, Y. N. 2016b, Sol. Phys., 291, 859

Zimovets, I. V., \& Struminsky, A. B. 2010, Sol. Phys., 263, 163

Zimovets, I. V., \& Nakariakov, V. M. 2015, A\&A, 577, A4

Zucca, P., Carley, E. P., Bloomfield, D. S., \& Gallagher, P. T. 2014, A\&A, 564, A47 


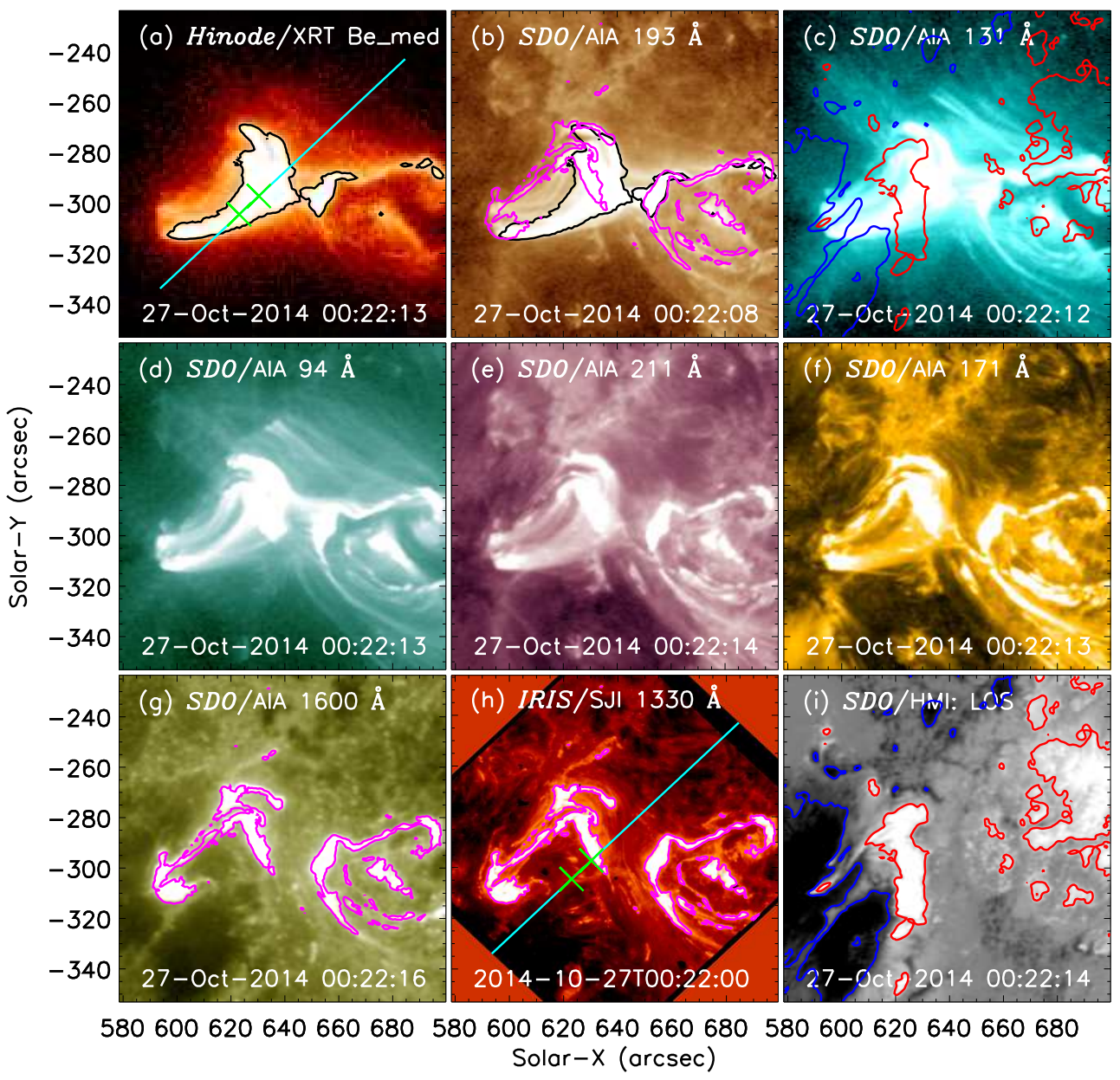

Fig. 1.- Simultaneous snapshots observed by Hinode/XRT, SDO/AIA, SDO/HMI and IRIS/SJI on 2014 October 27. The turquoise line marks the IRIS slit, and two short green lines are the bounds of the selected loop-top region. The black contours outline the SXR flare loop based on the XRT Be_med image with an intensity level of $700 \mathrm{DN}$, and the purple contours show the flare ribbons observed in SJI $1330 \AA$ image with an intensity level of $400 \mathrm{DN}$. The red and blue contours represent the positive and negative fields at the scale of $\pm 600 \mathrm{G}$ from HMI LOS magnetogram. A logarithmic scale is used in the XRT, AIA and SJI images. 


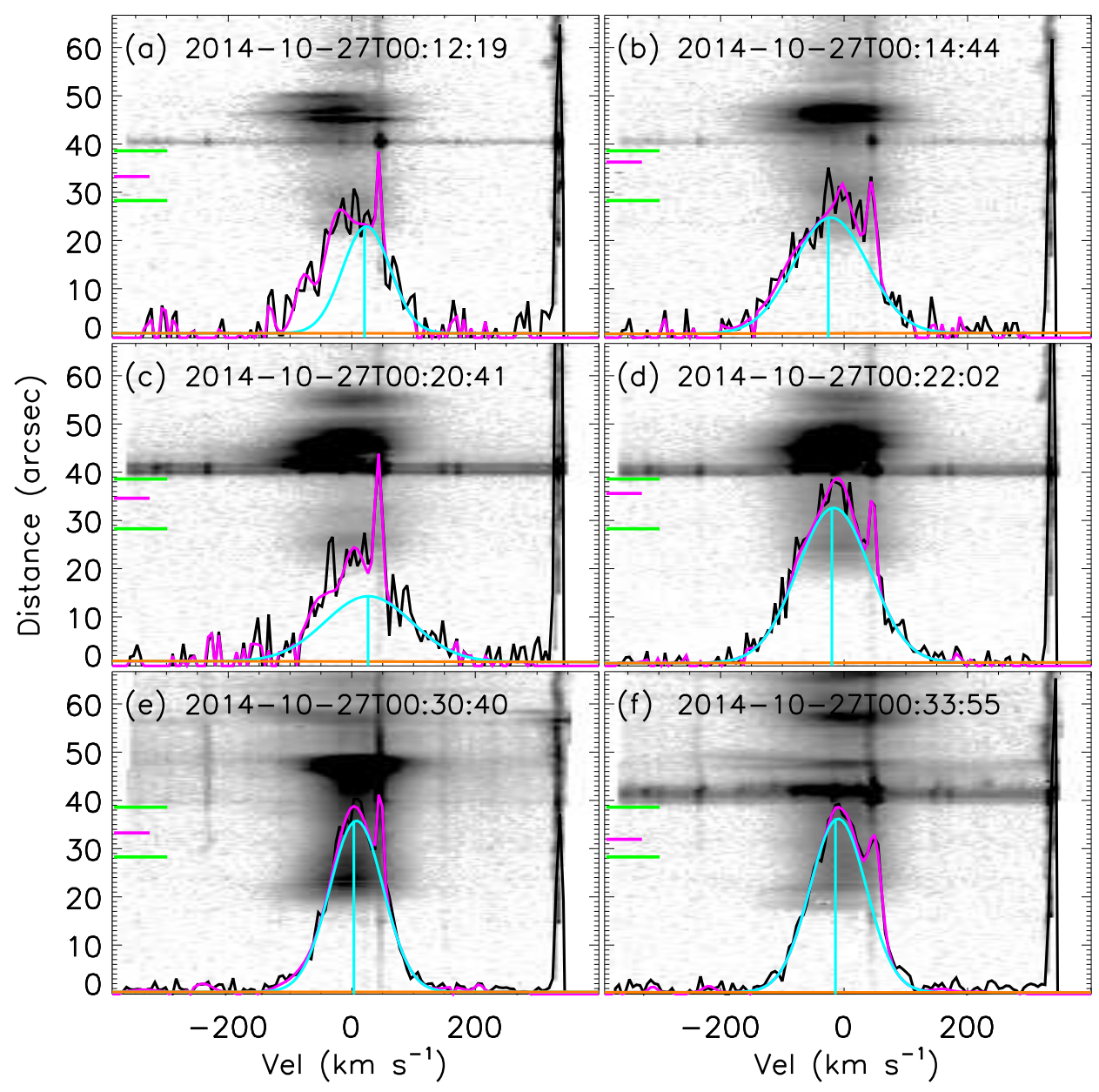

Fig. 2.- IRIS spectra and their fitting results at 'O I' window. Two short green lines are the bounds of the selected loop-top region. The black profile is the observed IRIS spectrum at the slit position marked by the short purple line. The purple profile gives the multi-Gaussian fitting with a linear background (marked by the orange line), and the turquoise profile is the extracted Fe XXI $1354.09 \AA$ line. The turquoise vertical line indicates the fitting line center of Fe Xxi $1354.09 \AA$. 


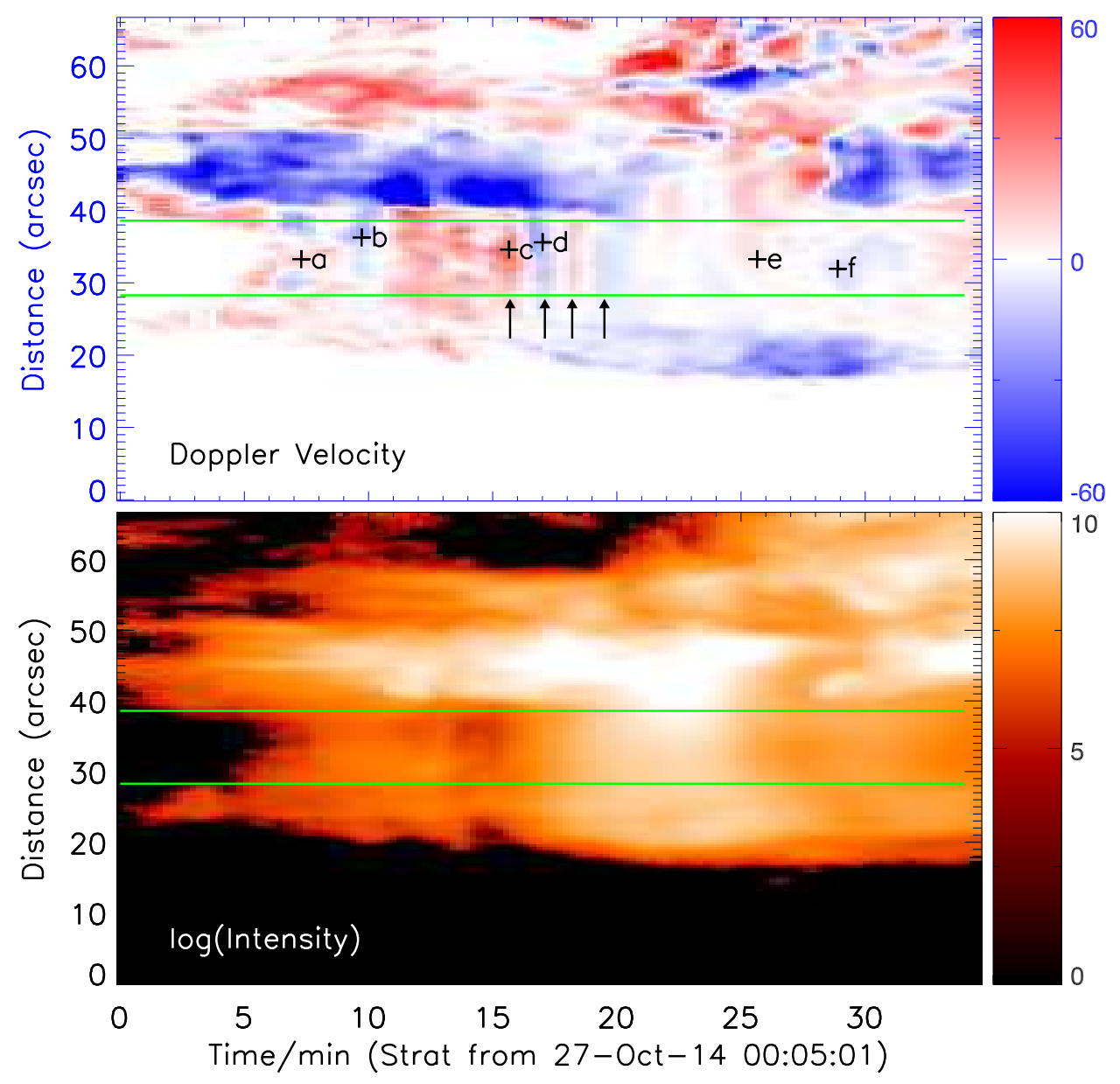

Fig. 3.- Upper: The space-time image of Doppler shift at Fe XXI 1354.09 A. Lower: The spacetime image of line-integrated intensity with a logarithmic brightness scale. Two green lines are the bounds of the selected loop-top region. The pluses ('+') mark the positions where the flare spectra are shown in Figure 2, 


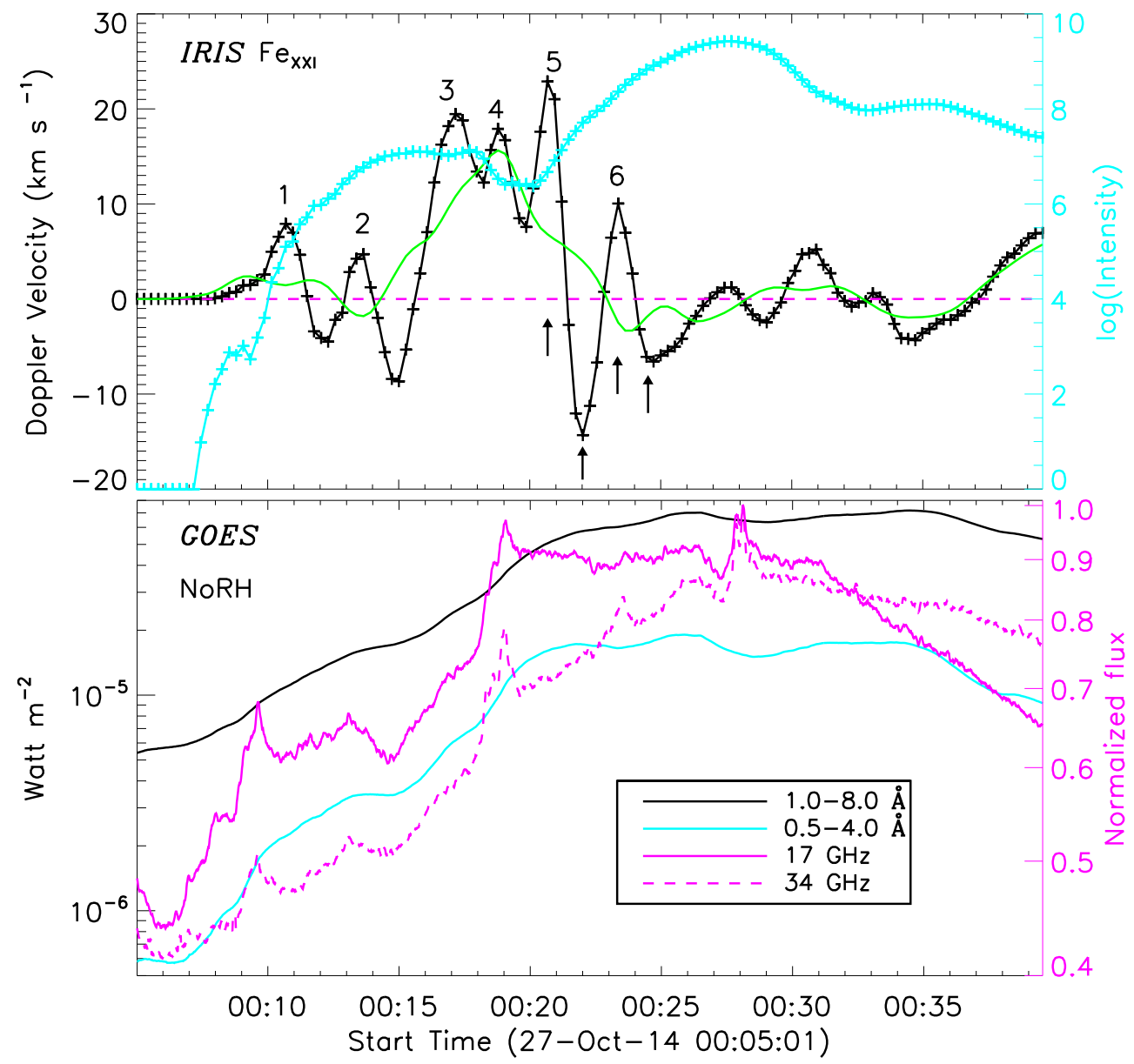

Fig. 4.- Upper: Temporal evolutions of Doppler shift (black) and line-integrated intensity (turquoise) at Fe Xxi $1354.09 \AA$. The green profile represents the background emission from Doppler shift. The oscillatory peaks are labeled with the number ticks. The purple dashed line marks the zero velocity, and the arrows mark the same time in Figure 3 , Lower: GOES SXR light curves at 1.0-8.0 $\AA$ (black) and 0.5-4.0 $\AA$ (turquoise), and normalized fluxes at NoRH $17 \mathrm{GHz}$ (solid purple) and $34 \mathrm{GHz}$ (dashed purple). 


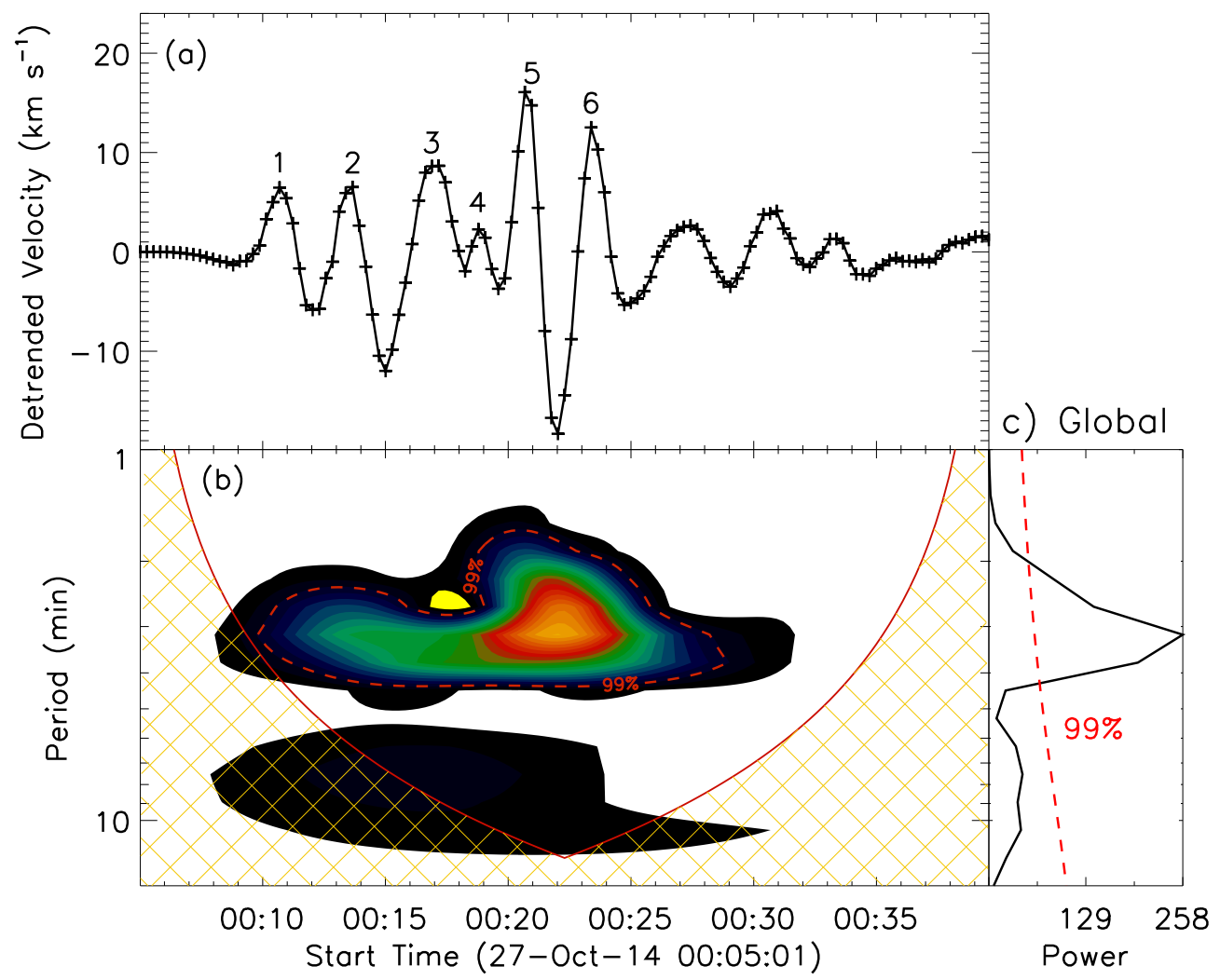

Fig. 5.- Panel (a): Temporal evolution of the detrended Doppler shift at Fe Xxi $1354.09 \AA$. Panel (b) and (c): Wavelet power spectrum and global power of the detrended Doppler shift. The dashed lines indicate a significance level of $99 \%$. 


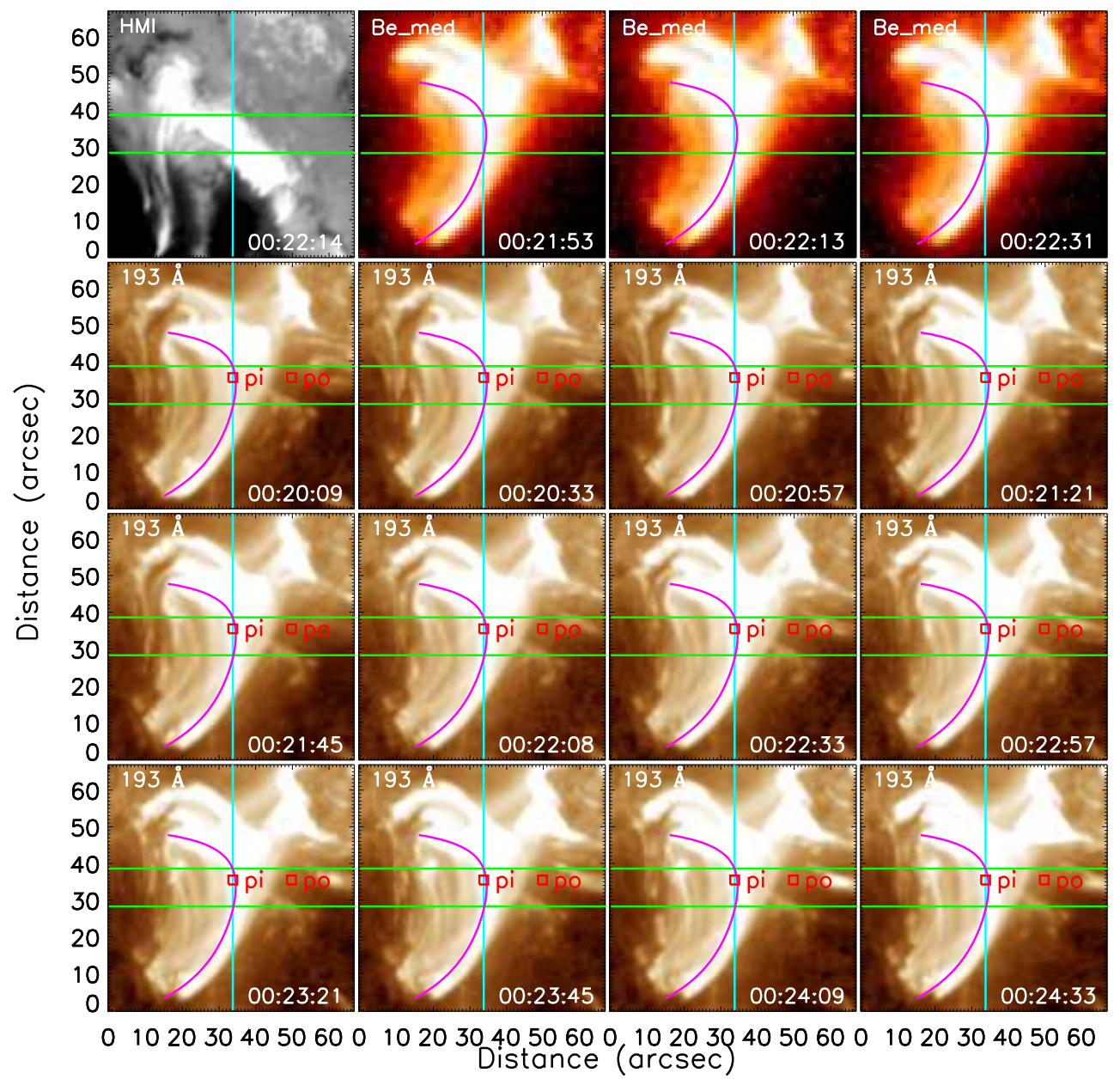

Fig. 6. - Snapshots along IRIS slit direction observed by HMI, XRT, and AIA, respectively. Two green lines are the bounds of the selected loop-top region, and the turquoise line marks the IRIS spectral slit. The purple line indicates the SXR loop. The red boxes outline the region $\left(2.4^{\prime \prime} \times 2.4^{\prime \prime}\right)$ used to do the DEM analysis in Figure 7 . The scale levels of magnetogram are $\pm 1000 \mathrm{G}$, and the XRT and AIA images are displayed on a logarithmic brightness scale. 


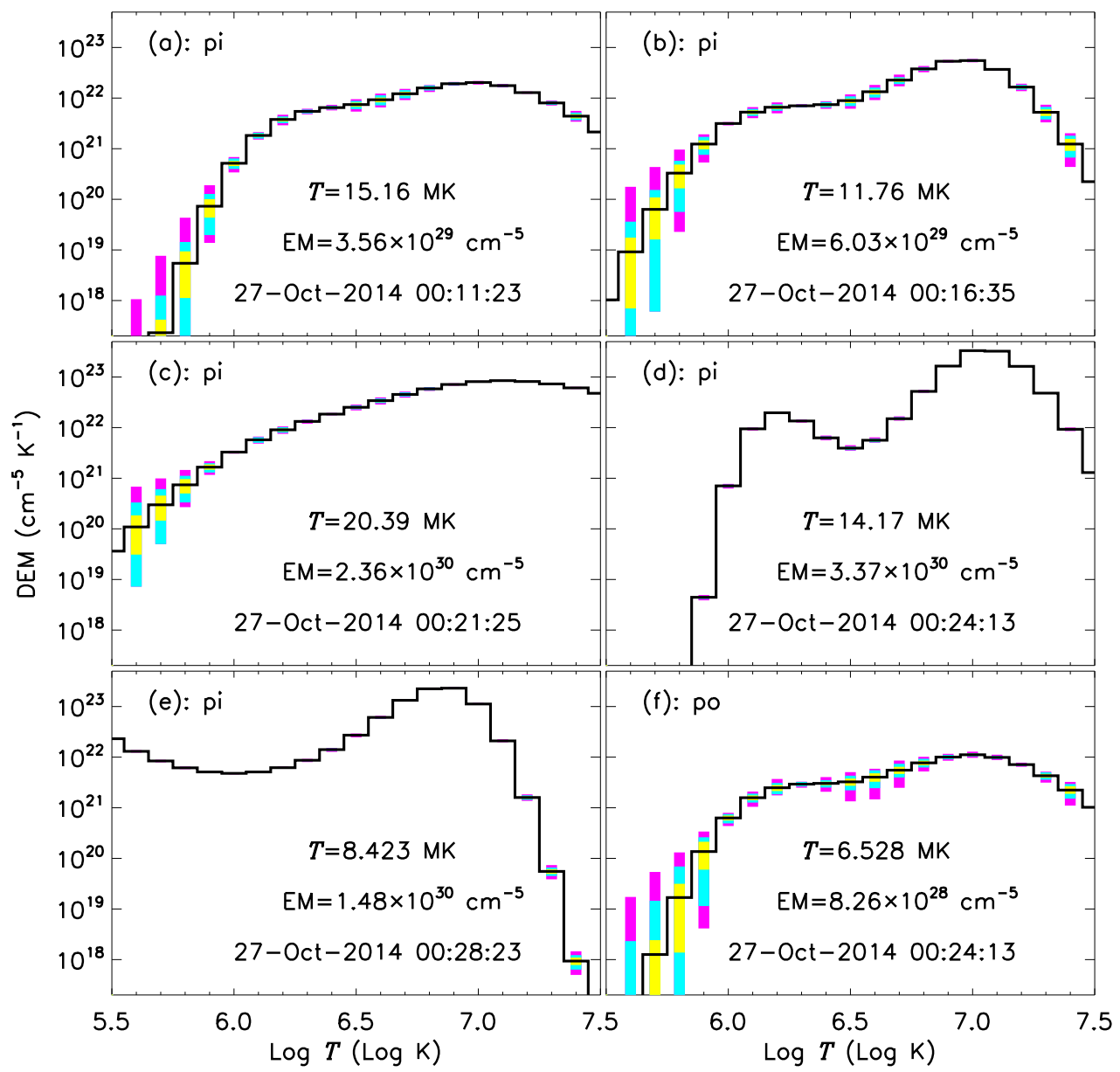

Fig. 7.- DEM profiles in the flare loop top (a-e) and background (f) regions, which are outlined by the small red boxes in Figure 6. The black profile shows the best-fitted DEM curve from AIA EUV observations. The yellow rectangles represent the regions that contains $50 \%$ of the MC solutions. The turquoise rectangles, above and below the yellow rectangles, and the yellow rectangles compose the regions that cover $80 \%$ of the MC solutions. All the colored rectangles form the regions which contain $95 \%$ of the MC solutions. The mean temperature, EM and observed time are given in each panel. 


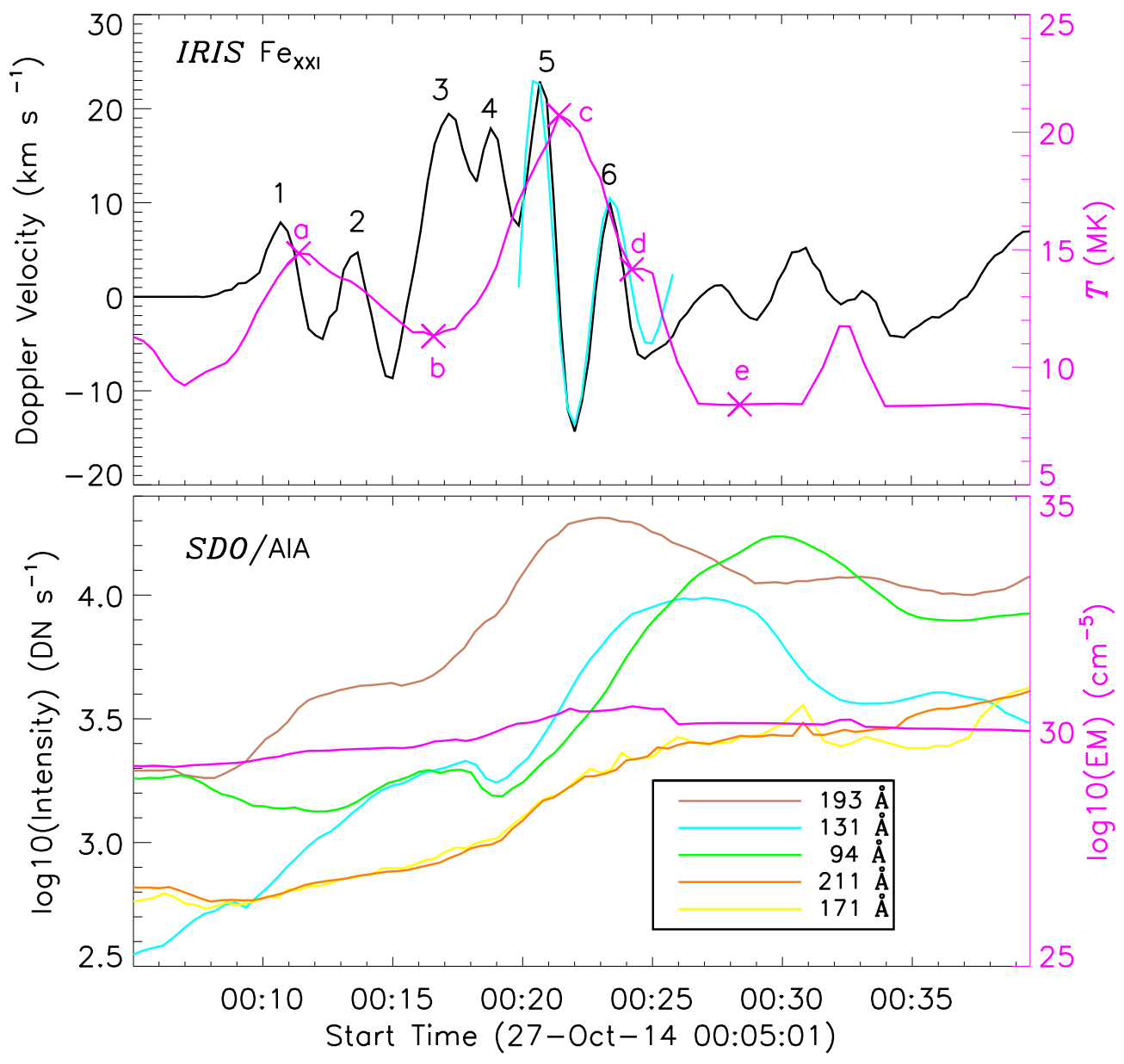

Fig. 8.- Upper: Time series of Doppler shift at Fe xxi $1354.09 \AA$ and temperature (purple) in the flare loop-top region. The turquoise line gives the best fit for the peaks ' 5 ' and ' 6 '. The crosses (' $\times$ ') indicate the time to display the DEM analysis results in Figure 7 . Lower: SDO/AIA light curves at $193 \AA$ (brown), $131 \AA$ (turquoise), $94 \AA$ (green), $211 \AA$ (orange), and $171 \AA$ (yellow). The purple profile is the temporal variations of EM in the flare loop-top region. 


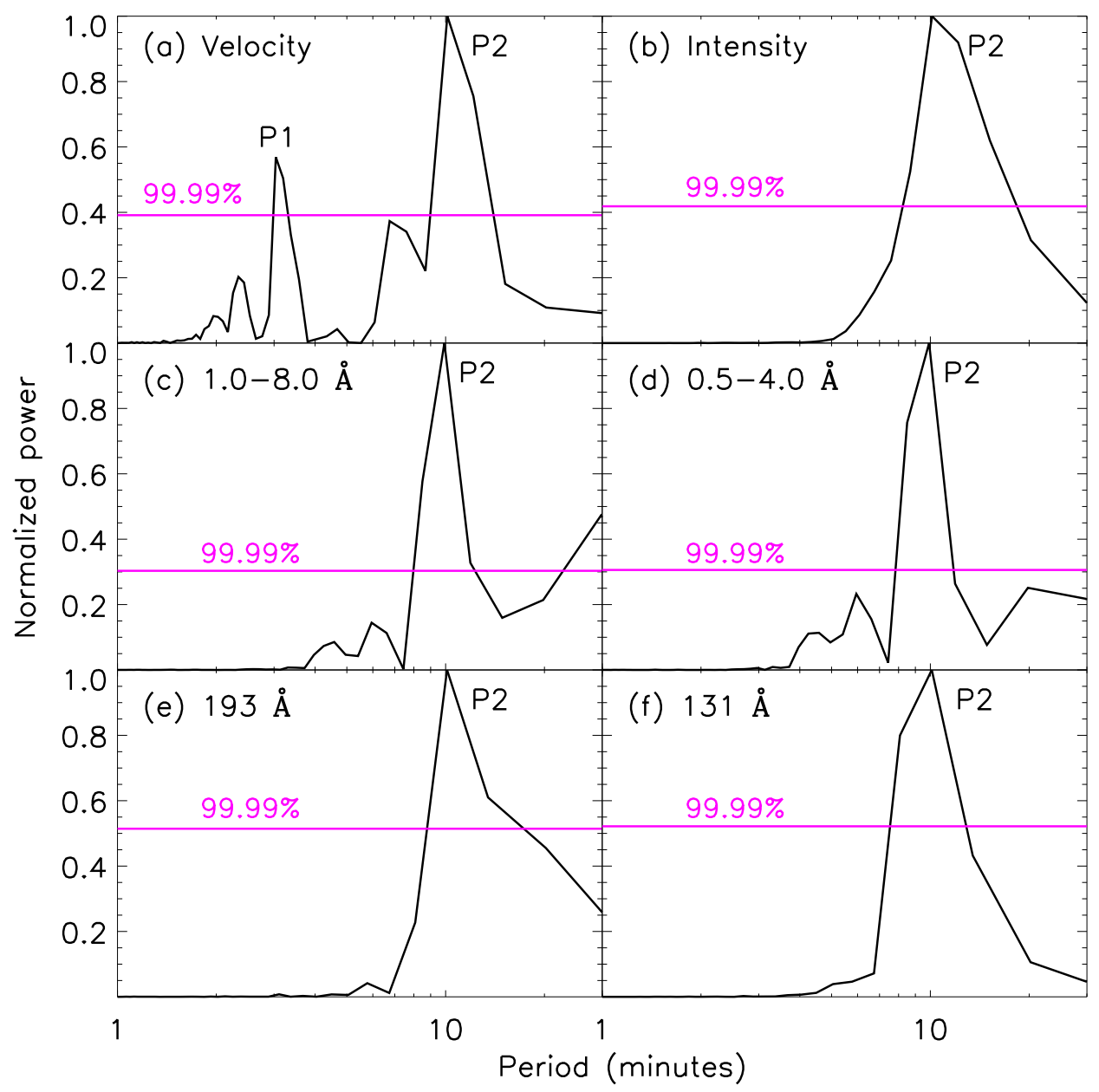

Fig. 9.- Normalized Fourier power spectra of the detrended time series from IRIS Fe XXI $1354.09 \AA$ (a, b), GOES SXR fluxes (c, d), and AIA EUV light curves (e, f). A horizontal purple line in each panel indicates the $99.99 \%$ confidence level. 